\title{
Morfologia de nectários em Leguminosae senso lato em áreas de caatinga no Brasil
}

\author{
Yanna Melo ${ }^{1,2,4}$, Elisabeth Córdula², Silvia R. Machado ${ }^{3}$ e Marccus Alves ${ }^{2}$
}

Recebido em 14/01/2010. Aceito em 13/10/2010

\begin{abstract}
RESUMO - (Morfologia de nectários em Leguminosae senso lato em áreas de caatinga no Brasil). Nectários extraflorais (Nefs) são glândulas secretoras de néctar encontradas em diversas espécies de Angiospermas, inclusive Leguminosae. Essas estruturas podem se apresentar sob diferentes formas (elevados, embebidos; com ou sem estipe; cores distintas) e posições nas plantas (na raque, no pecíolo), sendo essas características relevantes aos estudos de taxonomia e sistemática. Este trabalho analisou a diversidade morfológica dos Nefs em Leguminosae de uma área prioritária para a conservação da caatinga no Estado de Pernambuco. As 35 espécies de Legumionsae estudadas foram coletadas no Município de Mirandiba, no semi-árido Pernambucano, e submetidas às técnicas usuais para análise e descrição morfológica. Entre essas espécies, foram caracterizados Nefs com origem primária (não substitutivos) e secundária (substitutivos). Dois diferentes tipos com onze formatos distintos foram encontrados entre elas. Uma grande variedade de localização, coloração, projeção, e dimensão foram registradas aqui. Uma chave de identificação e ilustrações foram elaboradas também. Os dados apresentados aqui ampliam o número de espécies estudadas com Nefs para a família, e também confirmam a importância taxonômica e ecológica dessas estruturas para os legumes da região do semi-árido do Brasil.
\end{abstract}

Palavras-chave: Glândulas, morfologia, néctar, sistemática

ABSTRACT - (Nectary morphology of Leguminosae senso lato in areas of dry seasonal forest in Brazil). Extrafloral nectaries (Efns) are nectar secreting glands found in many species of Angiosperms, including Leguminosae. These structures have various forms (elevated, embedded; stalk present or not; different colors) and positions on the plants (on the rachis, on the petiole), and these characteristics are relevant to taxonomy and morphology studies. This work analyses the morphological diversity of Efns in Leguminosae from a priority conservation area of caatinga in Pernambuco state. The 35 Leguminosae species studied were collected in Mirandiba municipality, a semi-arid region of Pernambuco and submitted to the usual techniques of anatomy and morphology analyses. Among these species, Efns with primary (non-substitutive) and secondary (substitutive) origin were characterized. Two different kinds and eleven distinct Efns forms were found. A large variety of placement, color, shape, and size are reported here. An identification key and illustrations are also provided. Our data increased the number of species studied with Efns in the family, and also confirm the taxonomic and ecological importance of this structure for legumes from the semi-arid region of Brazil.

Key words: Glands, morphology, nectar, systematics

\section{Introdução}

Nectários extraflorais (Nefs) são bastante diversos quanto à morfologia e localização, e amplamente distribuídos em representantes de 113 famílias de Angiospermas (Bentley \& Elias 1983; Díaz-Castelazo et al. (2005); Keeler 2010). Muitos autores têm ressaltado a importância das variações nas características dessas glândulas como ferramenta eficaz para estudos de sistemática em diferentes categorias taxonômicas, especialmente na família Leguminosae (Bhattacharyya \& Marheshawari 1970a, 1970b; Metcalfe \& Chalk 1979; Leitão et al. 2002; Bortoluzzi et al. 2007; Morim \& Barroso 2007).

A variabilidade morfológica (interna e externa) dos Nefs, incluindo os de Leguminosae, foi ressaltada por autores em ambientes distintos como a Amazônia, a Caatinga e o Cerrado (Oliveira \& Leitão-Filho 1987; Lewis \& Owen 1989; Morellato \& Oliveira 1991; Conceição et al. 2008; Machado et al. 2008; Melo et al. 2010).

As Leguminosae apresentam distribuição cosmopolita, ocupando vários tipos de ambientes (Lewis et al. 2005). Na caatinga é a família de plantas com maior diversidade estando representada por cerca de 300 espécies, das quais 144 são endêmicas (Queiroz 2002; 2006a; 2006b). Além disso, vem sendo descrita como a maior família de Angiospermas com Nefs no mundo (Keeler 2010).

Desse modo, objetiva-se caracterizar morfologicamente os nectários (incluindo a análise de secreção) de Leguminosae ocorrentes em áreas de caatinga no Município de Mirandiba - PE, bem como construir uma chave de identificação a partir das informações adquiridas e informar acerca dos elementos de fauna que coletam o néctar nas espécies estudadas. Os dados aqui apresentados buscam ainda fornecer informações que auxiliem na circunscrição de táxons de Leguminosae e contribuam para o estudo de nectários no ecossistema caatinga.

\section{Material e métodos}

A área de estudo selecionada é uma das indicadas por Silva et al. (2004) como prioritária para o estudo científico e potencial conservação da flora. Localiza-se no município de Mirandiba, Pernambuco $\left(8^{\circ} 12^{\prime} \mathrm{S} 38^{\circ} 32^{\prime} \mathrm{W}\right)$ e possui aproximadamente $800 \mathrm{Km}^{2}$ de extensão. Está inserida no semi-árido pernambucano a cerca de $500 \mathrm{~km}$ de distância do Recife, na microrregião Salgueiro, no limite norte da ecorregião da Depressão Sertaneja Meridional (Velloso et al. 2002). O clima é quente, tipo estepe, com precipitação média anual de $431,8 \mathrm{~mm}$ e temperatura média anual de $27^{\circ} \mathrm{C}$ (Parahyba et al. 1998; Beltrão et al. 2005).

Foram realizadas seis coletas de campo entre outubro de 2006 e março de 2008, distribuídas na estação seca e chuvosa. Todos os representantes de

\footnotetext{
Universidade Federal de Pernambuco, Programa de Pós-Graduação em Biologia Vegetal, Recife, PE, Brasil

2 Universidade Federal de Pernambuco, Centro de Ciências Biológicas, Departamento de Botânica, Laboratório de Morfo-Taxonomia Vegetal, Recife, PE, Brasil

3 Universidade Estadual Paulista Júlio de Mesquita Filho, Instituto de Biociências, Departamento de Botânica, Laboratório de Morfologia Vegetal, Botucatu, SP, Brasil

4 Autor para correspondência: yannabotany@yahoo.com.br
} 
Leguminosas registrados para a região (Córdula et al. 2008) foram coletados (no modo de varredura da área) e analisados quanto à ocorrência de Nefs. As amostras dos Nefs foram retiradas de indivíduos de $5-10$ populações distintas localizadas em áreas de caatinga no Município de Mirandiba Em média 30 Nefs foram coletados por indivíduo, do total de três a cinco indivíduos de cada espécie por população.

Durante o período de coleta foram realizadas, ainda, visitas a outras áreas de Caatinga em Pernambuco (Municípios de Buíque, Veturosa e Serra Talhada) e Paraíba (Municípios de Boa Vista, Monteiro, Serra Branca e Sumé). Estas visitas objetivaram confirmar a ocorrência e características macroscópicas dos Nefs das espécies em estudo em populações distintas. Paralelamente foi realizado um levantamento nos principais herbários de Pernambuco (IPA, PEUFR e UFP) com o mesmo objetivo.

Amostras das espécies estudadas (ramos vegetativo e reprodutivo) foram identificadas através de bibliografia específica diversa e depositadas no Herbário UFP (Tabela 1). Os ramos com nectários foram fixados em formaldeído-ácido acético-álcool etílico 50\% (Kraus \& Arduim 1997) para análise de morfologia externa com extração de dados (comprimento, largura, diâmetro da região secretora) ou glutaraldeído $2,5 \%$ em tampão fosfato 0,1M, pH 7,3 para observação em Microscopia Eletrônica de Varredura (MEV) (Haddad et al. 1998). Secções (transversais e longitudinais) dos nectários foram submetidas ao reagente de Fehling para detectar a presença de açúcar redutor (Kraus \& Arduim 1997). A terminologia adotada para o formato e ornamentação dos Nefs seguiu Elias (1983) e Machado et al. (2008) com modificações propostas, como a inclusão de formato. Com base nos trabalhos de Vogel (1997) e Díaz-Castelazo et al. (2005) também foram adotados os termos Nefs substitutivos para estruturas que comumente apresentam outra função nas plantas (estípulas, pedicelo floral e tricomas), mas desenvolveram o potencial produtor e secretor de néctar; e não substitutivos para as glândulas com a caracterização e função típica de Nefs.

Elementos da fauna (formigas) associada, sempre que encontrados visitando os Nefs, foram capturados sempre que encontrados e fixados em FAA 50 para posterior identificação (por especialistas do departamento de Ecologia Vegetal da Universidade Federal de Pernambuco)

\section{Resultados}

Das 82 espécies (17 endêmicas da caatinga) registradas de Leguminosae em Mirandiba (Córdula et al. 2008), 35 (43\%) possuem Nefs. Dentre as espécies com Nefs, nove estão no grupo das endêmicas da caatinga e quatro têm poucos registros para o Estado de Pernambuco (Córdula et al. 2008). A subfamília Caesalpinioideae está representada na área de estudo por 23 espécies das quais $15(65 \%)$ tem Nefs, Mimosoideae com 24, sendo 17 (71\%) com Nefs e Papilionoideae com 35 espécies, das quais $3(8,5 \%)$ possuem Nefs (Tab.1).

As áreas de caatinga selecionadas para coleta variaram quanto aos aspectos vegetacionais indo da caatinga arbustiva esparsa a caatinga arbórea densa. Além disso, as espécies estudadas apresentaram variações de hábito (herbáceas, subarbustos, subarbustos aquáticos, arbustos e árvores) que reincidiram de uma área para outra.

Quanto à caracterização morfológica dos nectários, estes podem ser substitutivos ou não substitutivos; e apresentamse sob dois tipos básicos: estruturados, quando o tecido se diferencia para formar a glândula, e não estruturado, quando não há diferenciação. Foram reconhecidos onze formatos distintos (Tabela 2): embebido em fenda (Fig.1A-B), tricoma glândular (Fig.1C-D), elevado-calicióide (Fig.1EF), embebido-côncavo (Fig.2A-C), elevado-digitiforme (Fig.2D-E), elevado-globoso-côncavo (Fig.2F-G), elevadocôncavo(Fig.3A-B), elevado-plano (Fig.3C-D), incluso-côn- cavo (Fig.3E-F), elevado-estipuliforme (Fig.3G-H), glândula discóide (Fig.3I). Os Nefs podem ser estipitados (Fig.1E-F), não estipitados (Fig.2A-B, 3A-D) e levemente projetados ou sésseis (Fig.2F-G, 3G-H). Localizam-se em diferentes regiões das folhas e/ou inflorescências. Tanto a coloração quanto as dimensões são variáveis entre os tipos aqui catalogados (Tab.2), e variáveis entre si durante a maturação. Os caracteres observados nos Nefs estudados se mantiveram constantes em todas as populações acompanhadas. Todos os caracteres analisados estão resumidos na Tabela 2.

Todos os nectários apresentaram reação positiva ao teste de Fehling para detectar açúcar redutor, confirmando assim sua atividade secretora de néctar.

Dentre as espécies de Caesalpinioideae os gêneros $\mathrm{Li}$ bidibia e Poincianella foram os únicos a apresentar Nefs substitutivos (Tab.2). Libidibia ferrea (Mart ex Tul.) L.P. Queiroz var. ferrea (Caesalpinioideae) é a única espécie com Nefs não estruturados, os quais aparecem como máculas (possíveis de observar a olho desarmado) onde ocorre o rompimento do tecido secretor e acúmulo de néctar (observado a olho desarmado). Os mesmos são embebidos (inseridos no tecido do órgão ou constituídos pelo próprio tecido), formam fenda, são sésseis e de coloração verde. Localizam-se próximo a inserção do pedicelo com o botão floral e possuem em média 1,0 mm de comprimento (Fig.1A-B). Todas as demais espécies estudadas apresentam Nefs estruturados com morfologia e origem diversas.

Poincianella bracteosa Tul. e P. gardneriana Benth. (Caesalpinioideae) possuem Nefs sob a forma de tricomas glandulares nas folhas, os mesmos são estipitados, marrons e possuem em média de 0,07-0,2 mm de compr. (Fig.1CD). Os nectários de P. bracteosa e P. gardneriana secretam néctar suficiente para ser visto a olho desarmado, assim como a própria glândula é facilmente observada.

Os representantes de Chamaecrista Moench (Caesalpinioideae) estudados têm Nefs elevado-calicióides, estipitados, localizados na região mediana do pecíolo, de coloração verde e com média de 1,0-2,0 mm de compr. (Fig.1E-F). Chamaecrista repens (Vogel) H.S. Irwin \& Barneby difere das outras espécies do gênero por apresentar um a dois Nefs por pecíolo; e ter o estipe espesso, podendo atingir até a dimensão da cabeça secretora. Em Bauhinia cheilantha (Bong.) Steud (Caesalpinioideae) os Nefs também são elevado-calicióides, estipitados, verdes e com em média $1 \mathrm{~mm}$ de compr. No entanto, estão localizados na base do pecíolo e encobertos pelo par de estípulas.

Em Hymenaea courbaril L. (Caesalpinioideae) foram encontrados Nefs embebido-côncavos, sésseis, verdes, localizados nos folíolos e com 0,2-0,3 mm de compr. (Fig.2AB). A espécie apresentou ainda nectários florais (Nfs) que co-ocorrem com os Nefs. Estes se localizam nas sépalas e têm morfologia semelhante aos extraflorais aqui apresentados (Fig.2C). Embora não representem o foco central deste estudo os nectários florais e $H$. courbaril foram abordados e ilustrados para confirmação dos dados apresentados. 
Tabela 1. Lista das espécies de Leguminosae com Nefs coletadas em Mirandiba, PE. Material testemunho: EC = Elisabeth Córdula, KP = Katarina Pinheiro, YM = Yanna Melo.

\begin{tabular}{|c|c|}
\hline Espécies & Material Testemunho \\
\hline \multicolumn{2}{|l|}{ Caesalpinioideae } \\
\hline Bauhinia cheilantha (Bong.) Steud & EC et al. 252 \\
\hline Chamaecrista calycioides (DC. ex Collad.) Greene. & EC et al. 298 \\
\hline Chamaecrista duckeana (P. Bezerra \& Afr. Fernandes) H.S. Irwin \& Barneby & EC et al. 233 \\
\hline Chamaecrista pilosa (L.) Greene var. luxurians (Benth.) H.S. Irwin \& Barneby. & EC et al. 55 \\
\hline Chamaecrista repens (Vogel) H.S. Irwin \& Barneby & KР 479 \\
\hline Hymenaea courbaril L. & EC et al. 345 \\
\hline Libidibia ferrea (Mart. ex. Tul.) L.P. Queiroz var. ferrea & EC et al. 244 \\
\hline Poincianella bracteosa (Tul.) L.P. Queiroz & EC et al. 277 \\
\hline Poincianella gardneriana (Benth.) L.P. Queiroz & EC et al. 253 \\
\hline Senna macranthera (Coll.) H.S. Irwin \& Barneby var. pudibunda (Benth.) H.S. Irwin \& Barneby. & EC et al. 305 \\
\hline Senna obtusifolia (L.) H.S. Irwin \& Barneby & YM et al. 149 \\
\hline Senna occidentalis (L.) Link & EC et al. 296 \\
\hline Senna splendida (Vogel) H.S. Irwin \& Barneby var. gloriosa H.S. Irwin \& Barneby & EC et al. 271 \\
\hline Senna trachypus (Benth.) H.S. Irwin \& Barneby & EC et al. 273 \\
\hline Senna uniflora (Mill.) H.S. Irwin \& Barneby & YM et al. 149 \\
\hline \multicolumn{2}{|l|}{ Mimosoideae } \\
\hline Anadenanthera colubrina (Vell.) Brenan & EC et al. 206 \\
\hline Chloroleucon dumosum (Benth.) G.P. Lewis & KP et al. 249 \\
\hline Chloroleucon foliolosum (Benth.) G.P. Lewis & EC et al. 210 \\
\hline Desmanthus pernambucanus (L.) Thell. & YM et al. 280 \\
\hline Enterolobium contortisiliquum (Vell.) Morong. & EC et al. 178 \\
\hline Inga vera Willd. & EC et al. 340 \\
\hline Neptunia plena (L.) Benth. & YM et al. 161 \\
\hline Parapiptadenia zehntneri (Harms) M.P. Lima \& H.C. Lima & EC et al. 203 \\
\hline Parapiptadenia aff. zehntneri (Harms) M.P. Lima \& H.C. Lima & EC et al. 356 \\
\hline Piptadenia stipulacea (Benth.) Ducke & YM et al. 183 \\
\hline Piptadenia viridiflora (Kunth.) Benth. & EC et al. 330 \\
\hline Pithecellobium diversifolium Benth. & EC et al. 208 \\
\hline Pityrocarpa moniliformis (Benth.) Luckow \& R.W. Jobson & EC et al. 223 \\
\hline Prosopis juliflora (Sw.) DC. & YM et al. 273 \\
\hline Senegalia piauhiensis (Benth.) A. Bocage \& L.P. Queiroz. & EC et al. 212 \\
\hline Senegalia polyphylla (DC.) Britton \& Rose & EC et al. 355 \\
\hline Senegalia riparia (Kunth.) Britton \& Rose & EC et al. 190 \\
\hline \multicolumn{2}{|l|}{ Papilionoideae } \\
\hline Crotalaria incana $\mathrm{L}$. & EC et al. 54 \\
\hline Erythrina velutina Willd. & YM et al. 279 \\
\hline Rhynchosia minima (L.) DC. & EC et al. 36 \\
\hline
\end{tabular}




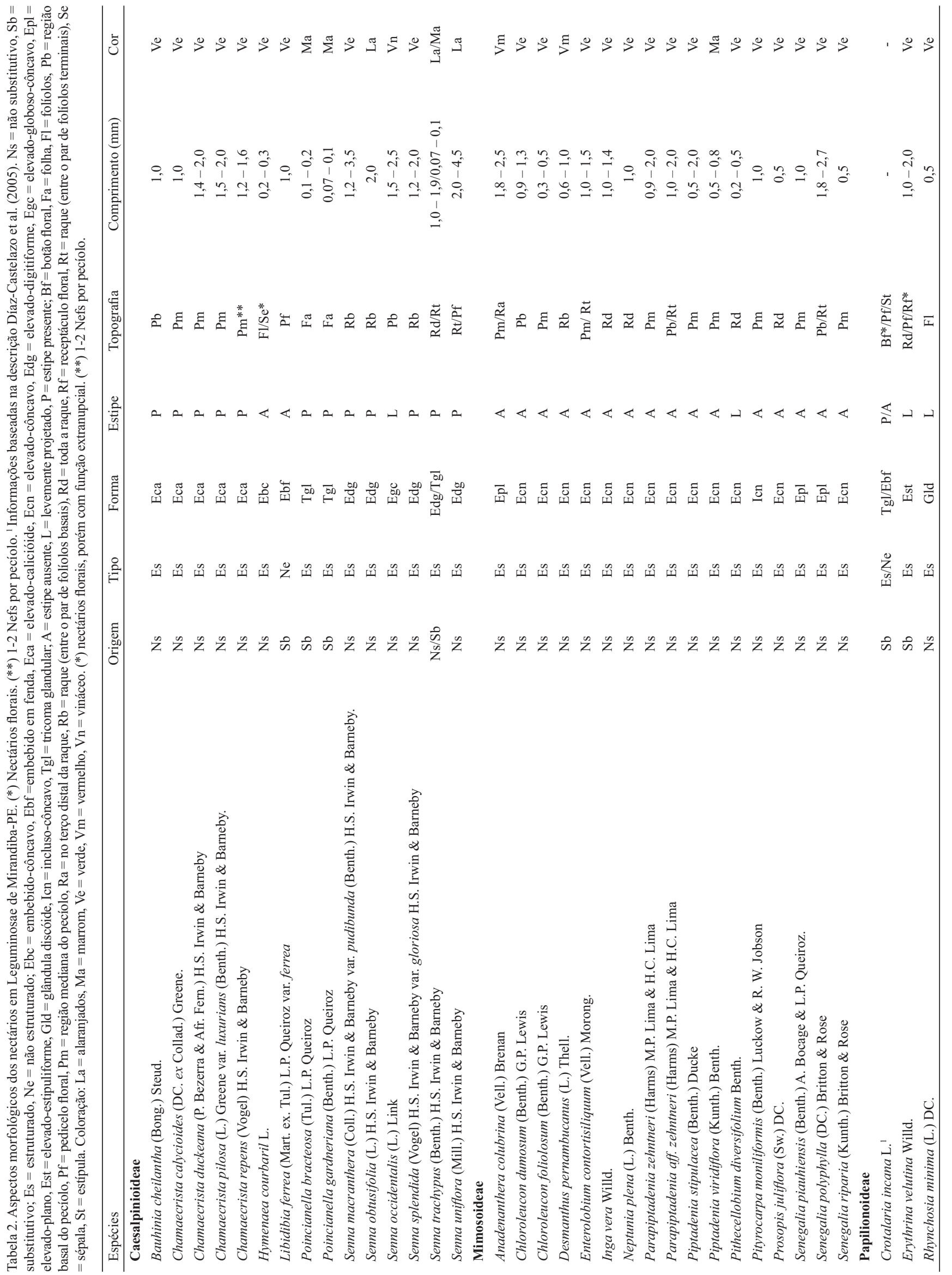



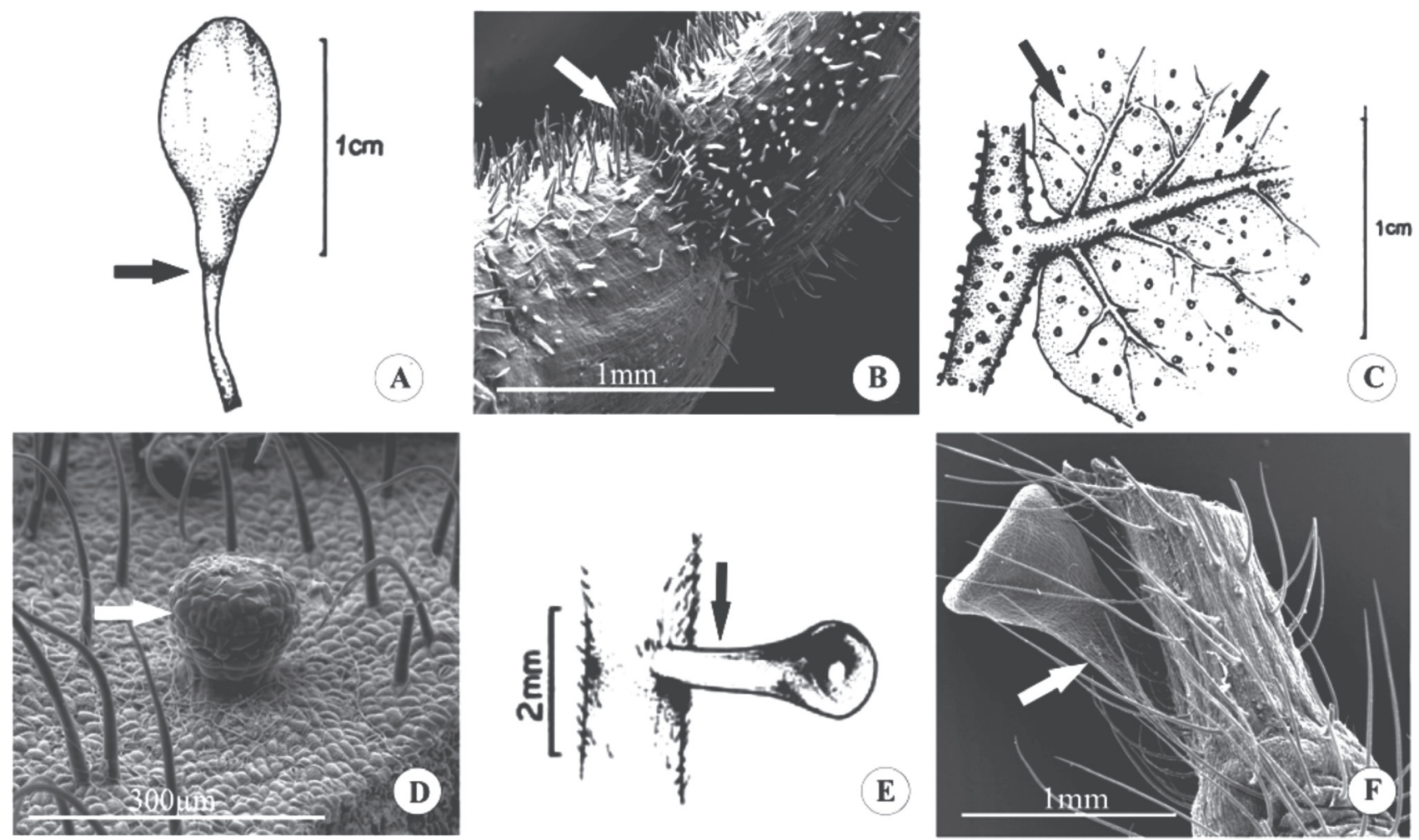

Figura 1. Nectários extraflorais (Nefs) em espécies de Leguminosae de Mirandiba-PE. A-B Libidibia ferrea (Mart ex. Tul) LP. Queiroz var. ferrea. (A) Ilustração demonstrando a mácula secretora de néctar que se apresenta no pedicelo floral. (B) Fotomicrografia eletrônica de varredura (MEV) do nectário extrafloral, seta branca apontando a fenda secretora do Nef. C-D Poincianella bracteosa (Tul) LP. Queiroz. (C) Ilustração mostrando a distruibuição dos nectários extraflorais por toda a raque e folíolo. (D) MEV da lâmina foliar (face abaxial) apontando o Nef (seta branca). E-F Chamaecrista pilosa (L) Greene var. luxurians (Benth) HS. Irwin \& Barneby. (E) Ilustração do Nef (seta apontando o estipe). (F) MEV do Nef preso ao ramo vegetativo (raque), seta apontando o estipe.

Das seis espécies de Senna Mill. (Caesalpinioideae) encontradas em Mirandiba, cinco possuem Nefs elevadodigitiformes (Fig.2D-E) e uma, Senna occidentalis (L.) Link, apresenta Nefs elevado-globoso-côncavos (Fig.2FG). Em Senna trachypus (Benth.) H.S. Irwin \& Barneby, além dos Nefs não substitutivos, são observados nectários substitutivos na forma de tricomas glandulares por toda a raque (Tab.2). Os Nefs elevado-digitiformes observados nas espécies de Senna podem ser estipitados ou levemente projetados, e estar localizados na região basal do pecíolo, no pedicelo floral ou na raque, variam de $1,0-4,5 \mathrm{~mm}$ de comprimento e tem coloração diversa (Tab.2).

Nas Mimosoideae, todos os Nefs foram não substitutivos. O formato predominante é o elevado-côncavo (Fig.3AB), entretanto Anadenanthera colubrina (Vell.) Brenan, Senegalia piauhiensis (Benth.) A. Bocage \& L.P. Queiroz e Senegalia polyphylla (DC.) Britton \& Rose apresentam Nefs elevado-planos (Fig.3C-D). Em Pytirocarpa moniliformis (Benth.) Luckow \& R. W. Jobson são incluso-côncavos (Fig.3E-F), que apesar de semelhante ao elevado-côncavo, difere por ser parcialmente inserido no órgão em que está localizado. Todos são sésseis a levemente projetados e com coloração variando de verde, vermelho a marrom. Podem estar localizados no pecíolo e na raque e com $0,2-2,7 \mathrm{~mm}$ de comprimento.

Crotalaria incana L., Erythrina velutina Willd. e Rhynchosia minima (L.) DC. foram os únicos representantes com Nefs encontrados na subfamília Papilionoideae. Os Nefs são substitutivos em $C$. incana e E.velutina, mas $R$. minima possui Nefs não substitutivos. Os Nefs de $C$. incana e R. minima (Fig.3I) foram descritos por outros autores em trabalho anterior e todas as informações corroboram com as análises feitas no presente estudo.

Em Erythrina velutina, os Nefs são elevado-estipuliformes e com poro secretor ventral, ocorrendo aos pares na raque e no pedicelo. São levemente projetados, verdes e variam de 1,0-2,0 mm de comprimento (Fig.3G-H). Ocorre ainda um par de nectários florais localizado no receptáculo floral, com estrutura similar aos Nefs encontrados (Tab.2). Esses nectários, embora sendo florais, foram registrados aqui porque apresentaram atividade secretora antes da antese sendo, portanto, extranupciais.

A diversidade dos Nefs observada permitiu a elaboração da seguinte chave de identificação visando o reconhecimento ou diagnóstico das espécies em estádio estéril: 

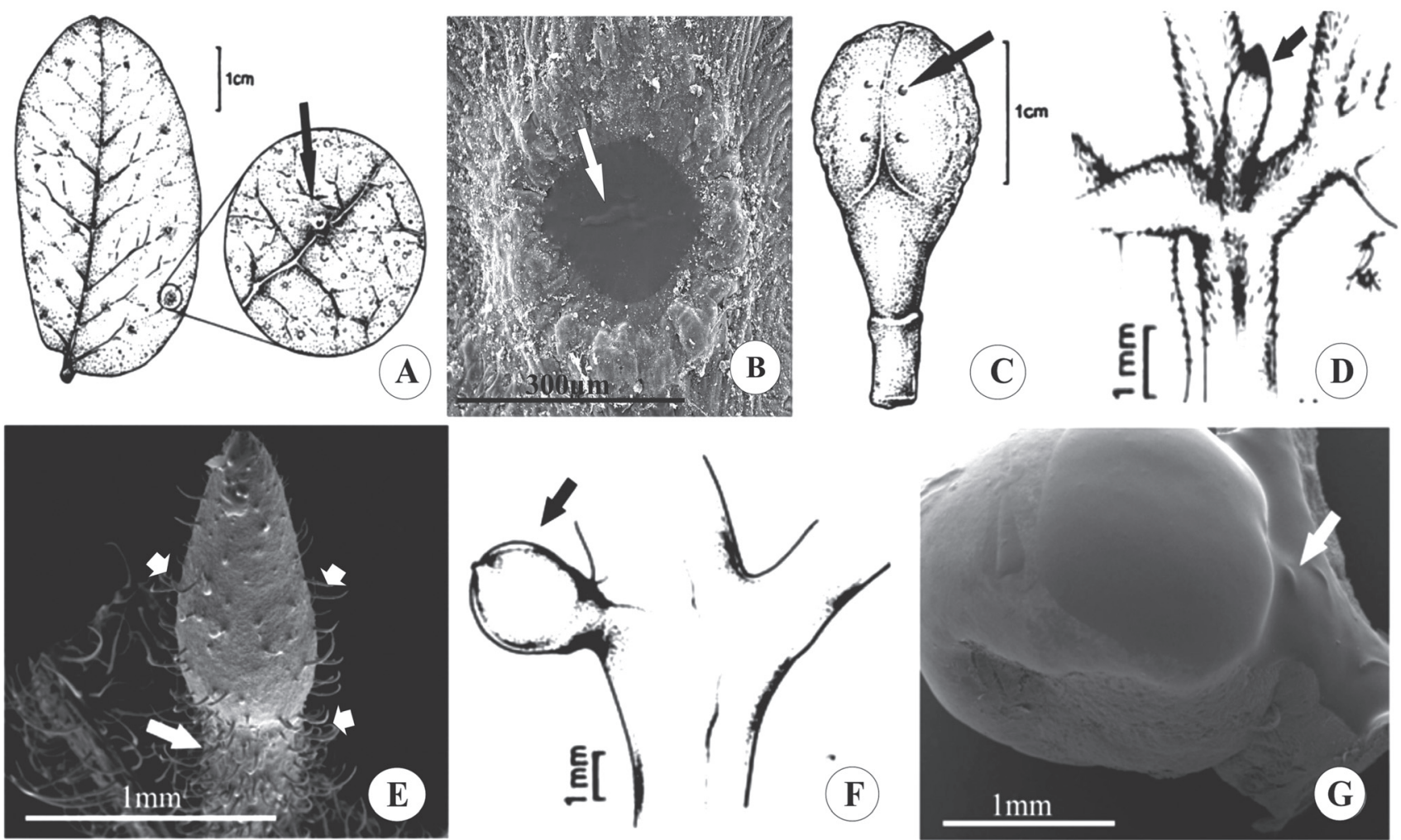

Figura 2. Nectários em espécies de Leguminosae de Mirandiba-PE. (*Nectários florais [Nfs]). A-C Hymenaea courbaril L. (A) Ilustração demonstrando a distribuição dos Nefs nos folíolos (seta). (B) Fotomicrografia eletrônica de varredura (MEV) do Nef observe a região central (seta branca). (C) Ilustração do botão floral a seta indica o Nf* localizado nas sépalas. D-E Senna splendida (Vogel) HS. Irwin \& Barneby var. gloriosa HS. Irwin \& Barneby. (D) Ilustração do Nef observe a região secretora indicada pela seta. (E) MEV mostrando o Nef fixo à raque (ramo), observe os tricomas distribuidos por todo o Nef (seta menor) e o estipe (seta maior). F-G Senna occidentalis (L) Link. (F) Ilustração do Nef (seta preta) disposto no pecíolo. (G) MEV do Nef disposto no pecíolo, seta branca apontando a leve projeção nectário.

Chave de identificação dicotômica das espécies de Leguminosae de Mirandiba, com base nos nectários extraflorais (Nefs)

1. Nefs exclusivamente substitutivos 2

2. Nefs exclusivamente não estruturados, no formato embebido em fenda 15. Libidibia ferrea var. ferrea

2 '.Nefs estruturados e/ou não estruturados, com formatos distintos. .3

3. Nefs estruturados sob a forma de tricomas glandulares e não estruturados no formato embebido em fenda no mesmo indivíduo

9. Crotalaria incana

$3^{\prime}$.Nefs exclusivamente estruturados com outros formatos.....

4. Nefs sob a forma de tricomas glandulares, com 0,07-0,2 mm compr.

23. Poincianella bracteosa $\mathrm{e}$

24. P. gardneriana

$4^{\prime}$.Nefs no formato elevado-estipuliforme, com 1,0 - 2,0 mm de compr.

12. Erythrina velutina

1 '.Nefs não substitutivos e/ou substitutivo . .5

5. Nefs não substitutivos e substitutivos no mesmo indivíduo

34. Senna trachypus

5 '. Nefs exclusivamente não substitutivos

6

6. Nefs embebido-côncavos

13. Hymenaea courbaril

6'.Nefs incluso-côncavos, glândulas discóides ou elevados com formatos distintos

7. Nefs incluso-côncavos 7

7'.Nefs como glândulas discóides ou elevados com formatos distinto

22. Pityrocarpa moniliformis

8. Nefs em forma de glândulas discóides dispostas na lâmina foliar.

8'.Nefs elevados com forma e localização variadas

26. Rhynchosia minima

9. Nefs com distribuição (quantidade), localização e formas diversas............................................................. 10

10. Nefs exclusivamente na região mediana do pecíolo 
11. Nefs um a dois por pecíolo no mesmo individuo

$11^{\prime}$.Nefs exclusivamente um por pecíolo

12. Nefs elevado-calicióides

13. Nefs com $1,0 \mathrm{~mm}$ de compr.

3. Chamaecrista calycioides

13'.Nefs 1,4-2,0 mm de compr. .... 4. Chamaecrista duckeana e 5. Chamaecrista pilosa var. luxurians

$12^{\prime}$.Nefs com formatos diversos

14. Nefs elevado-planos

..27. Senegalia piauhiensis

14'.Nefs elevado-côncavos

15. Nefs marrons

20. Piptadenia viridiflora

$15^{\prime}$.Nefs verdes

16. Nefs com cavidade central ampla

19. Piptadenia stipulacea

$16^{\prime}$.Nefs com cavidade central reduzida

17. Nefs com $0,3-0,5 \mathrm{~mm}$ de compr

8. Chloroleucon foliolosum $\mathrm{e}$

29. Senegalia riparia

$17^{\prime}$.Nefs com $0,9-2,0 \mathrm{~mm}$ de compr.

18. Parapiptadenia zehntneri

$10^{\prime}$.Nefs em regiões diversas da folha e/ou no pedicelo floral 18

18. Nefs exclusivamente na região basal do pecíolo

19. Nefs elevado-calicióides

2. Bauhinia cheilantha

19'. Nefs com formatos distintos 20

20. Nefs elevado-globoso-côncavos

32. Senna occidentalis

20'.Nefs elevado-côncavos.

7. Chloroleucon dumosum

$18^{\prime}$.Nefs na raque ou no pedicelo floral

21. Nefs exclusivamente entre o par de folíolos basais da raque

22. Nefs elevado-côncavos .22

22'. Nefs elevado-digitiformes

23. Nefs alaranjados com $2,0 \mathrm{~mm}$ de compr.

23'.Nefs verdes com $1,2-3,5 \mathrm{~mm}$ de compr.

31. Senna obtusifolia

24. Nefs com estipe estreito não atingindo a espessura da cabeça secretora

33. Senna

splendida var. gloriosa

24'.Nefs com estipe largo atingindo a espessura da cabeça secretora

30. Senna

macranthera var. pudibunda

21'. Nefs em regiões distintas da raque e/ou no pedicelo floral.

25

25. Nefs entre o par de folíolos terminais da raque e no pedicelo floral no mesmo indivíduo

35. Senna uniflora

$25^{\prime}$.Nefs exclusivamente dispostos ao longo de toda a raque

26. Nefs elevado-côncavos, levemente projetados

21. Pithecellobium diversifolium

26’.Nefs elevado-côncavos, sésseis

27. Nefs com $0,5 \mathrm{~mm}$ de compr

25. Prosopis juliflora

$27^{\prime}$.Nefs $>1,0 \mathrm{~mm}$ de compr...... . .28

28. Nefs com cavidade central ampla

$28^{\prime}$.Nefs com cavidade central reduzida

14. Inga vera

16. Neptunia plena

9'.Nefs com distribuição regular (quantidade), localização especifica no pecíolo e na raque num mesmo indivíduo e formas distintas

29. Nefs na região basal do pecíolo e entre o par de folíolos terminais da raque...... 30 30. Nefs elevado-planos

28. Senegalia polyphylla

30 '.Nefs elevado-côncavos 17. Parapiptadenia aff. zehntneri

$29^{\prime}$.Nefs na região mediana do pecíolo e em regiões distintas da raque no mesmo indivíduo. 31

31. Nefs elevado-côncavos, na região mediana do pecíolo e exclusivamente entre o par de folíolos terminais da raque.

11. Enterolobium contortisiliquum

31'.Nefs elevado-planos, na região mediana do pecíolo e no terço distal da raque

1. Anadenanthera colubrina 

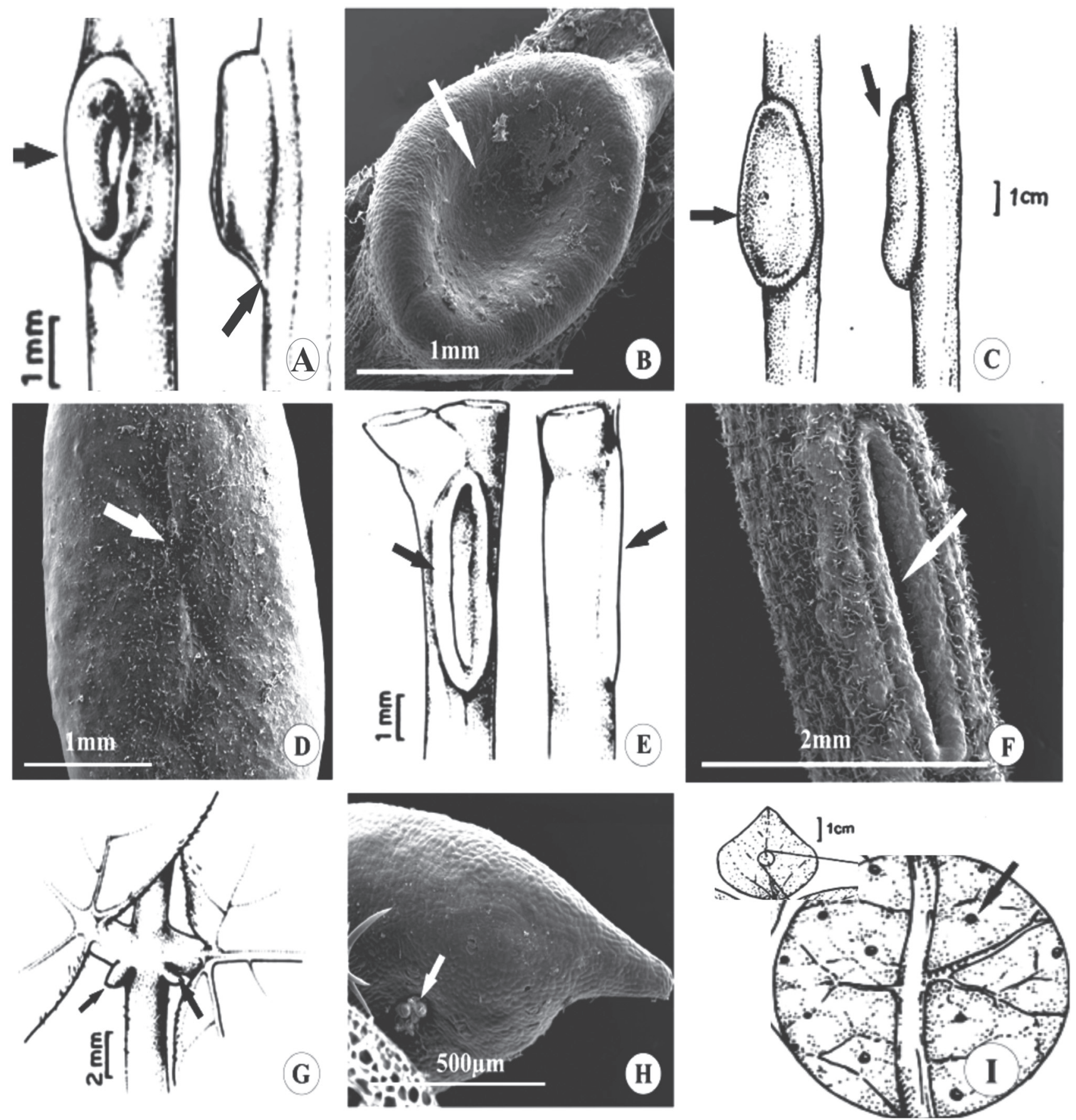

Figura 3. Nectários extraflorais (Nefs) em Leguminosae (Mirandiba-PE). A-B. Piptadenia stipulacea (Benth.) Ducke. (A) Ilustração do Nef observe a região secretora (seta menor) e área de inserção do nectário no pecíolo com ausência de estipe (seta maior). (B) Fotomicrografia eletrônica de varredura (MEV) mostrando a região secretora do Nef (seta). C-D. Anadenanthera colubrina (Vell.) Brenan. (C) Ilustração mostrando a vista frontal e lateral do Nef plano (setas), observe a inserção do Nef (sem estipe) no ramo (vista lateral). (D) MEV do Nef mostrando a região secretora (seta branca). E-F. Pityrocarpa moniliformis (Benth.) Luckow \& R.W. Jobson. (E) Ilustração do Nef mostrando sua inserção no pecíolo (setas). (F) MEV do Nef mostrando a região secretora (seta branca). G-H. Erythrina velutina Willd. (G) Ilustração dos Nefs na raque foliar (setas). (H) MEV do Nef mostrando região secretora com tricomas glandulares (seta branca). I. Rhynchosia mínima (L.) DC.Ilustrando o aspecto puntiforme dos Nefs (glândulas discóides) distribuídos por todo o folíolo (seta). 
Além do aspecto taxonômico, os Nefs das espécies estudadas também apresentaram associações com formigas dos seguintes gêneros: Camponotus (Camponotini-Formicidae), Cephalotes (Cephalotini-Myrmicinae), Crematogaster (Crematogastrini-Myrmicinae) e Dorymyrmex (Dolichoderini-Dolichoderinae). As formigas visitavam os Nefs e coletavam o néctar durante o período de atividade destes. Diferentes espécies de formiga podiam ser observadas coletando néctar em uma mesma espécie de planta.

\section{Discussão}

Dentre as 35 espécies de Leguminosae estudadas, 22 têm Nefs sendo descritos pela primeira vez. Esse número eleva para 1047 a lista de espécies de Leguminosae com Nefs publicada por Keeler 2010. Além disso, dentre as espécies de Mirandiba que são endêmicas da caatinga (Córdula et al. 2008) mais de 50\% apresentaram Nefs, o que reforça a relevância dessas estruturas para a caracterização das espécies em que ocorrem.

O percentual de espécies com Nefs nas subfamílias Mimosoideae e Caesalpinioideae demonstra a ampla distribuição dessas estruturas nas duas subfamílias, enquanto que em Papilionoideae o número de espécies com Nefs é menor. Diversos estudos acerca da distribuição dos Nefs em Leguminosae têm ressaltado essa condição (Elias 1983; Judd et al. 1999).

Os aspectos evolutivos e ecológicos podem ser a chave para o esclarecimento da distribuição dos Nefs em Leguminosae (Pascal et al.2000; Conceição et al. 2008). De acordo com Pascal et al. (2000) há uma forte relação evolutiva entre membros da subfamília Mimosoideae com outros da tribo Caesalpinieae que possuem Nefs semelhantes. Polhill et al. (1981) sugerem ainda que a associação com formigas é um sistema de defesa comum às subfamílias Caesalpinioideae e Mimosoideae, enquanto que as Papilionoideae são mais dependentes de defesas químicas, como alcalóides, isoflavonóides e outros.

É possível que a distribuição dos Nefs em Leguminosae esteja relacionada à evolução reprodutiva dos grupos (Polhil et al. 1981). Mimosoideae e Caesalpinioideae apresentam o maior número de representantes com Nefs e possuem flores com caracteres plesiomórficos cujos órgãos reprodutivos são mais expostos e susceptíveis às agressões ambientais (Polhil et al. 1981). Papilionoideae apresentam caracteres apomórficos, tendo seu aparato reprodutivo melhor protegido (Polhil et al. 1981), o que reduziria o investimento em recursos no desenvolvimento de Nefs.

Nectários extraflorais variam quanto ao tipo, forma, localização e outros caracteres, tornando-se uma ferramenta eficaz para a taxonomia (Keeler \& Kaul 1979; Bentley \& Elias 1983). Em Leguminosae já foram examinados Nefs elevados, tricomas glandulares, embebidos e outros, com localizações e aspectos morfológicos diversos (Elias 1983; Paiva \& Machado 2006; Machado et al. 2008). Os tipos já descritos para os Nefs de Leguminosae são compatíveis com a maioria dos encontrados neste estudo. Além disso, a constância dos caracteres morfológicos dos Nefs entre as populações avaliadas demonstra seu potencial para a taxonomia da família.

Os Nefs de Libidibia ferrea var. ferrea se enquadram no tipo amorfo ou não estruturado, próprios do tecido secretor que não se diferencia para originar um órgão definido. De acordo com Elias (1983) e Castro \& Machado (2006), estes Nefs assemelham-se a máculas superficiais onde apenas o néctar é visualizado. Nectários florais (Nfs) similares foram descritos para outras espécies de Leguminosae e de Lamiaceae e Melastomataceae (Vogel 1997). No entanto, a ocorrência de Nefs não estruturados em Leguminosas é rara (Díaz-Castelazo et al. 2005). Embora presentes no pedicelo floral esses nectários são considerados extraflorais, pois de acordo com Bentley \& Elias 1983 os nectários podem ser classificados como: extraflorais no caso dos que se distribuem nos ramos vegetativos (pedicelo floral, pecíolo e raque foliar) e florais para àqueles que se localizam diretamente na flor.

Geralmente, entre os táxons estudados, os mais relacionados filogeneticamente apresentam Nefs semelhantes quanto à forma como em Poincianella bracteosa, P. gardneriana que apresentam tricomas glandulares secretores de néctar. Porém, espécies de táxons distintos também podem apresentar Nefs semelhantes entre si como foi observado em Senna trachypus que também apresentaram tricomas glandulares secretores de néctar. Outros gêneros de Caesalpinioideae têm esse tipo de Nef como foi observado por Pascal et al. (2000) e Machado et al. (2008). De acordo com Metcalfe \& Chalk (1979) os tricomas glandulares nectaríferos ocorrem agrupados formando manchas. Esta informação não é corroborada com as observações aqui realizadas, pois em $P$. bracteosa e $P$. gardneriana os Nefs são esparsos e se distribuem por toda a lâmina foliar.

Segundo Pohil et al. (1981), a co-evolução envolvendo plantas e animais em função da defesa ou polinização possivelmente conduziu ao surgimento dos Nefs. Há registros de hidatódios que se transformaram em Nefs e de estruturas intermediários entre essas estruturas (Evert 2006). Esse mecanismo de substituição ou transformação tem permitido que diferentes estruturas, como os tricomas, as estípulas e os hidatódios, transformem-se anatomicamente (de acordo com a evolução e necessidade da espécie) e atuem como efetivos secretores de néctar (Vogel 1997; Evert 2006).

A história evolutiva dos nectários não estruturados, ainda não é clara. Alguns autores acreditam que este seja um caráter plesiomórfico e em contrapartida, há relatos da possível origem secundária dessas estruturas (Stein \& Tobe 1989; Renner 1993).

Nas espécies da subfamília Caesalpinioideae foram registrados Nefs estruturados e não estruturados; substitutivos e não substitutivos, sendo os Nefs substitutivos observados em Libidibia ferrea var. ferrea, Poincianella bracteosa, $P$. gardneriana e Senna trachypus; sendo que nesta última 
também são observados Nefs não substitutivos. Dessas, as três primeiras espécies já foram agrupadas dentro do gênero Caesalpinia, o que indica uma possível relação evolutiva para os Nefs destes táxons. Além disso, a presença de diferentes tipos de Nefs nas espécies da subfamília Caesalpinioideae, sugere que essas glândulas podem apresentar um papel evolutivo intermediário em Leguminosae. No entanto, as observações até então realizadas não oferecem subsídios suficientes para conclusões adicionais.

Os gêneros Bauhinia, Chamaecrista e Senna estão entre os mais representativos para a subfamília (Judd et al. 1999) e em todos estes são encontrados representantes com Nefs. Diversos estudos confirmam a ampla distribuição dos Nefs nos gêneros Chamaecrista e Senna, incluindo Senna occidentalis (= Cassia occidentalis) (Bhattacharyya \& Marheshwari 1970b; Francino et al. 2006; Keeler 2010; Queiroz 2008) inclusive com morfologia e localização semelhantes.

Das duas espécies de Bauhinia na região de estudo, Bauhinia acuruana Moric. e B. cheilantha (Córdula et al. 2008), apenas nesta última foram registrados Nefs. É comum a presença de Nefs nos membros de Bauhinia serie Cansenia na qual a espécie está inserida (Vaz \& Tozzi 2003; 2005). Os Nefs encontrados em $B$. cheilantha têm formato semelhante ao das espécies de Chamaecrista aqui abordadas.

Em outras espécies de Bauhinia foram observados Nefs com constituição morfológica diferente dos visualizados em B. cheilantha (Vaz \& Tozzi 2003, 2005; Machado et al. 2008). A ocorrência de diferentes tipos de Nefs em espécies de um mesmo gênero parece estar relacionada à evolução independente destas complexas estruturas. Por outro lado, também ocorrem Nefs morfologicamente semelhantes, entre espécies de diferentes táxons, mas em grupos evolutivamente mais relacionados (Pascal et al. 2000).

Os Nefs embebidos, como os encontrados em Hymenaea courbaril, foram registrados pela primeira vez em Leonardoxa africana, Leguminosae (Elias 1980). Paiva \& Machado (2006) registraram este mesmo tipo em Hymenaea stigonocarpa. Embora, embebidos nos órgãos onde ocorrem, esses Nefs se enquadram na categoria dos nectários estruturados porque apresentam diferenciação dos tecidos (Melo 2008). Hymenaea courbaril também possui nectários florais $(\mathrm{Nfs})$ embebidos, localizados nas sépalas que co-ocorrem com os Nefs. Nesses nectários, a secreção ocorre pela face abaxial na pré-antese, quando também foi observada a presença de formigas. Portanto, esses Nfs apresentam função extranupcial como na maioria dos Nefs (Castro \& Machado 2006). Quanto à localização, os nectários florais observados nas sépalas de $H$. courbaril se adequam ao termo circunfloral proposto por Delpino (1873) apud Schmid (1988), para aqueles presentes em partes florais, mas não necessariamente relacionados à polinização.

Os Nefs em espécies de Mimosoideae demonstraram similaridade estrutural e topográfica entre si. Os tipos de Nefs observados nas espécies de Mirandiba, também foram descritos em outros táxons de Mimosoideae (Pascal et al.
2000; Díaz-Castelazo et al. 2005; Machado et al. 2008). Ebinger et al. (2000) em sua revisão do gênero Acacia observaram a presença de Nefs semelhantes em morfologia e localização aos das Mimosoideae aqui tratadas.

Apesar das semelhanças entre os Nefs da subfamília Mimosoideae, cada táxon possui características próprias que permitem sua distinção. Os Nefs de Parapiptadenia aff. zehntneri e P. zehntneri distinguem-se apenas pela sua localização. Com base no caráter localização dos Nefs é possível que $P$. aff. zehntneri trate-se de uma variedade taxonômica de P. zehntneri.

A semelhança estrutural dos Nefs em gêneros distintos sugere uma provável convergência desse caráter, devido à origem múltipla dos Nefs (McKey 1989; Soltis et al. 2005).

Nos poucos representantes com Nefs registrados para Papilionoideae, em sua maioria, estes se constituem em Nefs substitutivos, ou seja, estípulas, tricomas, pedicelo ou outras estruturas presentes na planta que passaram a funcionar como Nefs (Lersten \& Brubaker 1987; Díaz-Castelazo et al. 2005).

A subfamília Papilionoideae apresentou o menor número de representantes com Nefs, corroborando informações prévias (Elias 1983). Os Nefs estipuliformes em Erythrina velutina também ocorrem em outras espécies do gênero Erytrina e em outros gêneros da subtribo Phaseoleae à qual a espécie pertence (Lersten \& Brubaker 1987). Crotalaria incana tem Nefs embebidos em fenda e tricomas glandulares, localizados na inserção das estípulas, do botão floral e do pedicelo floral como foi descrito por Díaz-Castelazo et al. 2005. Em Rhynchosia minima, os Nefs são glândulas discóides (Bhattacharyya \& Marheshwari 1970a), dispersas nos folíolos, levemente projetadas, verdes e com $0,5 \mathrm{~mm}$ de comprimento (Fig.3I).

Bhattacharyya \& Marheshawari (1970a) já haviam descrito os Nefs de Rhynchosia minima e os dados aqui corroboram as observações realizadas. Díaz-Castelazo et al. (2005) registraram a presença tricomas nectaríferos e Nefs não estruturados em outras espécies de Papilionoideae. No entanto, em E. velutina e R. minima isto não foi observado.

A presença de estruturas transformadas parece proporcionar a redução do custo energético na planta e possivelmente limita o investimento de recursos no desenvolvimento de uma nova estrutura (Díaz-Castelazo et al. 2005). É provável que a aquisição de nectários transformados conote um avanço evolutivo para as Leguminosae com Nefs. Além disso, estes Nefs se distribuem principalmente em Papilionoideae, grupo mais derivado da família (Polhil et al. 1981; Elias 1983; Lersten \& Brubaker 1987).

O papel ecológico dos Nefs também tem sido comprovado a partir da relação mutualística observada entre plantas com Nefs e formigas (Heads \& Lawton 1985; Oliveira \& Pie 1988; Santos \& Del-Claro 2001; Madureira \& Sobrinho 2002; Fernandes et al. 2005; Bovendorp 2009). As formigas visitantes dos Nefs estudados, já foram encontradas em diferentes grupos de plantas. Existem registros a cerca do potencial de defesa contra herbivoria para Camponotus sp., 
Cephalotes sp. e Crematogaster sp. (Oliveira \& Pie 1998; Santos \& Del-Claro 2001; Fernandes et al. 2005; Nishimura 2009). Possivelmente essas formigas atuem em defesa das espécies de Leguminosae estudadas, no entanto é importante observar que também há registros de formigas oportunistas que visitam os Nefs, mas não apresentam a relação de defesa (Kaminski et al. 2009).

Os dados obtidos revelam a diversidade estrutural e a ampla distribuição dos Nefs em representantes de Leguminosae na caatinga, com destaque especial para as espécies endêmicas que das 17 registradas no ecossistema, nove (53\%) foram coletadas em Mirandiba e em todas foi verificada a presença de Nefs. A diversidade estrutural observada nos Nefs permitiu a identificação da maioria das espécies estudadas (excetuandose somente os pares: Chamaecrista duckeana e C. pilosa var. luxurians; Poincianella bracteosa e P. gardneriana (Caesalpinioideae); Chloroleucon foliolosum e Senegalia riparia (Mimosoideae) que apresentaram Nefs idênticos). Isso destaca a relevância dos Nefs como caráter diagnóstico para as táxons em questão. Além disso, as associações observadas entre Nefs e formigas coletoras de néctar ressaltam o importante aspecto ecológico dos Nefs para a manutenção das plantas e sua interação com a fauna, bem como parte da diversidade biológica existente na caatinga de Mirandiba, reforçando a necessidade de sua conservação.

\section{Agradecimentos}

Os autores gostariam de agradecer ao $\mathrm{CNPq}$ pela concessão da bolsa de Pós-Graduação concedida a primeira autora, a Fundação o Boticário de Proteção à Natureza pelo Financiamento do Projeto 0743_20071 e a Fundação de Amparo à Pesquisa do Estado de São Paulo (FAPESP). A equipe do Laboratório de Morfo-Taxonomia Vegetal (MTV). A MSc. Laís Borges (Laboratório de Biologia Floral e Reprodutiva, da UFPE) pelas proveitosas discussões, aos membros do Laboratório de Ecologia pela identificação dos táxons associados. Também agradecemos as equipes técnicas do Laboratório de Morfologia Vegetal e do Centro de Microscopia Eletrônica (IB/UNESP/Botucatu).

\section{Referências bibliográficas}

Beltrão, B.A.; Mascarenhas, J.C.; Miranda, J.L.F.; Souza Jr., L.C.; Galvão, M.J.T.G. \& Pereira, S.N. 2005. Diagnóstico do Município de Mirandiba: Projeto Cadastro de Fontes de Abastecimento por Água Subterrânea. Recife, Serviço Geológico do Brasil (CPRM)/ Programa de Desenvolvimento Energético dos Estados e Municípios (PRODEEM).

Bentley, B. \& Elias, T.S. 1983. The Biology of Nectaries. New York, Columbia University Press.

Bhattacharyya, B. \& Marheshwari, J.K. 1970a. Studies on extrafloral nectaries of the Leguminales. I. Papilionaceae, with a discussion on the systematics of the Leguminales. Proceedings of the Indian Academy of Sciences 37B(1): 11-30.

Bhattacharyya, B. \& Marheshwari, J.K. 1970b. Studies on extrafloral nectaries of the Leguminales. II. The genus Cassia Linn. (Caesalpinaceae). Proceedings of the Indian Academy of Sciences 37B(1): 74-90.

Bortoluzzi, R.L.C.; Miotto, S.T.S \& Reis, A. 2007. Novos registros de Chamaecrista Moench e Senna Mill. (Leguminosae-CaesalpinioideaeCassieae) na flora sul-brasileira.Iheringia, Sér. Bot 62(1-2): 121-130.

Bovendorp, R.S. 2009. Herbivoria foliar em Hibiscus pernambucensis (Malavaceae), uma planta com Nectários Extraflorais: Quanto vale um recruta zero? Pp. 1-3. In: Machado, G.; Prado, P.I. \& Oliveira, A.A. (eds). Livro do curso de campo "Ecologia da Mata Atlântica”. São Paulo, Universidade de São Paulo.

Castro, M.M. \& Machado, S.R. 2006. Células e Tecidos Secretores. Pp. 179-203. In: Appezzato-da-Glória, B. \& Carmello-Guerreiro, S.M. (eds). Anatomia Vegetal. Viçosa, Universidade Federal de Viçosa.

Conceição, A.S.; Queiroz, L.P.; Lambert, S.M.; Pereira, A.C.S. \& Borba, E.L. 2008. Biosystematics of Chamaecrista sect. Absus subsect. Baseophyllum (Leguminosae-Caesalpinioideae) based on allozyme and morphometric analyses. Plant Systematics and Evolution 270(3): 183-207.

Córdula, E.; Queiroz, L.P. \& Alves, M. 2008. Diversidade e distribuição de Leguminosae em uma área prioritária para a conservação da Caatinga em Pernambuco. Rodriguésia 59(3): 597-602

Díaz-Castelazo, C.; Rico-Gray, V.; Ortega, F. \& Ángeles, G. 2005. Morphological and Secretory Characterization of Extrafloral Nectaries in Plants of Coastal Veracruz, Mexico. Annals of Botany 96(7): 1175-1189.

Ebinger, J.E.; Seigler, D.S. \& Clarke, H.D. 2000. Taxonomic Revision of South American species of the genus Acacia subgenus Acacia (Fabaceae: Mimosoideae). Systematic Botany 25(4): 588-617.

Elias, T.S. 1980. Foliar Nectaries of Unsual Structure in Leonardoxa Africana (Legumnosae), an African Obligate Myrmecophyte. American Journal of Botany 67(3): 423-425.

Elias, T.S. 1983. Extrafloral Nectaries: their structure and distribution. Pp 174-203. In: Bentley, B. \& Elias, T.S. (eds). The Biology of Nectaries. New York, Columbia University Press.

Evert, R.F. 2006. Esau's Plant Anatomy. Meristems, Cells, and Tissues of the Plant Body - Their Structure, Function, and Development. New Jersey, John Wiley \& Sons, Inc.

Fernandes, G.W.; Fagundes, M.; Greco, M.K.B.; Barbeitos, M.S. \& Santos, J.C. 2005. Ants and their effects on an insect herbivore community associated with the inflorescences of Byrsonima crassifolia (Linneaus) H.B.K. (Malpighiaceae). Revista Brasileira de Entomologia 49(2): 264-269.

Francino, D.M.T.; Sant'anna-Santos, B.F.; Silva, K.L.F.; Thadeo, M.; Meira, R.M.S.A. \& Azevedo, A.A. 2006. Anatomia foliar e caulinar de Chamaecrista trichopoda (Caesalpinioideae) e histoquímica do nectário extrafloral. Planta Daninha 24(4): 695-705.

Haddad, A.; Sesso, A.; Attias, M.; Farina, M.; Meirelles, M.N.; Silveira, M.; Benchimol, M.; Soares, M.J.; Barth, O.M.; Machado, R.D.; SoutoPadrón, T. \& Souza, W. 1998. Técnicas básicas de Microscopia Eletrônica aplicadas às Ciências Biológicas. Rio de Janeiro, Sociedade Brasileira de Microscopia Eletrônica.

Heads, P.A. \& Lawton, H. 1985. Bracken, ants and extrafloral nectaries. III. How insect herbivores avoid ant predation. Ecological Entomology 10(3): 29-42.

Judd, W.S.; Campbell, C.S.; Kellong, E.A. \& Stevens, P.F. 1999. Plant Systematics. A Phylogenetic Approach. Massachusetts, Sinauer Associates, Inc.

Kaminski, L.A.; Sendoya, S.F.; Freitas, A.V.L. \& Oliveira, P.S. 2009. Ecologia comportamental na interface formiga-planta-herbívoro: interações entre formigas e Lepdópteros. Oecologia Brasiliensis 13(1): 27-44

Keeler, K.H. \& Kaul, R.B. 1979. Morphology and distribution of petiolar nectarines in Ipomoea (Convolvulaceae). American Journal of Botany 66(8): 946-952.

Keeler, K.H. 2010. World list of Angiosperm species with extrafloral nectaries. http://www.biosci.unl.edu/emeriti/keeler/extrafloral/ worldlistfamilies.htm./ (Acesso em 14/09/2010).

Kraus, E.J. \& Arduim, M. 1997. Manual básico de métodos em Morfologia Vegetal. Rio de Janeiro, Editora da Universidade Rural do Rio de Janeiro.

Leitão, C.A.E.; Meira, R.M.S.A.; Azevedo, A.A. \& Araújo, J.M. 2002. Ontogenia dos nectários extraflorais de Triumffeta semitriloba (Tiliaceae). Planta Daninha 20(3): 343-351.

Lersten, N.R. \& Brubaker, C.L. 1987. Extrafloral nectaries in Leguminosae: Review and orginal observations in Erythrina and Mucuna (Papilionoideae; Phaseoleae). Bulletin of the Torrey Botanical Club 114(4): 437-447.

Lewis, G.P. \& Owen, P.E. 1989. Legumes of the Ilha de Maracá. Surrey, Royal Botanical Gardens. 
Lewis, G.P.; Schrire, B.; Machinder, B. \& Lock, M. 2005. Legumes of the World. Surrey, Royal Botanic Gardens

Machado, S.R.; Morellato, L.P.C.; Sajo, M.G. \& Oliveira, P.S. 2008. Morphological patterns of extrafloral nectaries in woody plant species of the Brazilian cerrado. Plant Biology 10(4): 1-14.

Madureira, M. \& Sobrinho, T.G. 2002. Evidência de mutualismo entre Qualea cordata (Vochysiaceae) e Cephalotes sp. (Hymenoptera: Formicidae). Academia Insecta 2(1): 1-4.

McKey, D. 1989. Interactions between ants and Leguminous plants. Monographs in Systematic Botany of Missouri Botanical Garden 29: 673-718.

Melo, Y. 2008. Diversidade de nectários extraflorais em Leguminosae em áreas de caatinga - PE. Dados não publicados. Dissertação. Recife, Universidade Federal de Pernambuco.

Melo, Y.; Machado, S.R. \& Alves, M. 2010. Anatomy of extrafloral nectaries in Fabaceae from dry-seasonal forest in Brazil. Botanical Journal of the Linnean Society 163(1): 87-98.

Metcalfe, C.R. \& Chalk, L. 1979. Anatomy of the Dicotyledons V.1. Oxford, Clarendon Press.

Morellato, L.P.C. \& Oliveira, P.S. 1991. Distribution of Extrafloral Nectaries in Different Vegetation Types of Amazonian Brazil. Flora 185(5): 33-38.

Morim, M.P. \& Barroso, G.M. 2007. Leguminosae arbustivas e arbóreas da Floresta Atlântica do Parque Nacional do Itatiaia, Sudeste do Brasil: subfamílias Caesalpinioideae e Mimosoideae. Rodriguésia 58(2): 423-468.

Nishimura, P.Y. 2009. Formigas ou esclerificação foliar: Quem irá proteger Hibiscus pernambucensis (Malvaceae) do ataque de herbívoros? In: G. Machado, P.I. Prado \& A.A. Oliveira (eds). Livro do curso de campo “Ecologia da Mata Atlântica”. São Paulo, Universidade de São Paulo.

Oliveira, P.S. \& Leitão-Filho, H.F. 1987. Extrafloral Nectaries: Their taxonomic Distribution and Abundance in the Woody Flora of Cerrado Vegetation in Southeast Brazil. Biotropica 19(2): 140-148.

Oliveira, P.S. \& Pie, M.R. 1998. Interaction Between Ants and Plants Bearing Extrafloral Nectaries in Cerrado Vegetation. Anais da Sociedade Entomológica do Brasil 27(2): 161-176.

Paiva, E.A.S. \& Machado, S.R. 2006. Ontogênese, anatomia e ultraestrutura dos nectários extraflorais de Hymenaea stigonocarpa Mart. ex Hayne (Fabaceae-Caesalpinioideae). Acta Botanica Brasilica 20(2): 471-482.

Parahyba, R.B.V.; Silva, F.H.B.B.; Araújo Filho, J.C.; Silva, F.B.R. \& Maia, J.L.T. 1998. Diagnóstico Ambiental do Município de Mirandiba: destaque antecipado do projeto de Zoneamento Agroecológico do Estado de Pernambuco. Recife, Embrapa.

Pascal, L.M.; Motte-Florac, E.F. \& McKey, D.B. 2000. Secretory structures on the leaf rachis of Caesalpinieae and Mimosoideae (Leguminosae) implications for the evolution of Nectary Glands. American Journal of Botany 87(3): 327-338.

Polhill, R.M.; Raven, P.H. \& Stirton, C.H. 1981. Evolution and Systematics of the Leguminosae. Pp: 1-26. In: Polhill, R.M. \& Raven, P.H. (eds)
Advances in Legumes systematic Part 1. Kew, Royal Botanic Gardens.

Queiroz, L.P. 2002. Distribuição das Leguminosae na Caatinga. Pp: 141153. In: Sampaio, E.V.S.B.; Giulietti, A.M.; Virgínio, J. \& GamarraRojas, C.F.L. (Eds). Vegetação \& Flora da Caatinga. Recife, Associação de Plantas do Nordeste - APNE, Centro de Informações sobre Plantas - CNIP.

Queiroz, L.P. 2006a. The Brazilian Caatinga: Phytogeographical Patterns Inferred from Distribution Data of the Leguminosae. Pp: 113-149. In: Pennington, R.T.; Lewis, G.P. \& Ratter, J.A. Neotropical Savanas and Seasonally Dry Forests. Plant Diversity, Biogeography and Conservation. London, CRC Press Taylor \& Francis Group.

Queiroz, L.P. 2006b. Flowering plants of the Brazilian semi-arid. Pp.4953. In: Queiroz, L.P.; Rapini, A. \& Giulietti, A.M. Towards greater knowledge of the Brazilian semi-arid biodiversity. Brasília, Ministério da Ciência e Tecnologia.

Queiroz, L.P. 2008. Leguminosas da Caatinga. Feira de Santana. Universidade Estadual de Feira de Santana, Associação de Plantas do Nordeste-APNE. Kew, Royal Botanic Gardens.

Renner, S.S. 1993. Phylogeny and classification of the Melastomataceae and Memecylaceae. Nordic Journal of Botany 13(5): 519-540.

Santos, J.C. \& Del-Claro, K. 2001. Interação entre formigas, herbívoros e nectários extraflorais em Tocoyena formosa (Cham. \& Schlechtd.) K. Schum. (Rubiaceae) na vegetação do cerrado. Revista Brasileira de Zoociências 3(1): 77-92.

Schmid, R. 1988. Reproductive Versus Extra-reproductive Nectaries Historical Perspective and Terminological Recommendations. The Botanical Review 54(2): 179-233.

Silva, J.M.C.; Tabarelli, M.; Fonseca, M.T. \& Lins, L.V. 2004. Biodiversidade da Caatinga: áreas e ações prioritárias para a conservação. Brasília, Ministério do Meio Ambiente (MMA).

Soltis, D.E.; Soltis, P.S.; Endress, P.K. \& Chase, M.W. 2005. Phylogeny and Evolution of Angiosperms. Massachusetts, Sinauer Associates, Inc. Publishers.

Stein, B.A. \& Tobe, H. 1989. Floral nectaries in Melastomataceae and their systematic and evolutionary implications. Annals of the Missouri Botanical Garden 76(2): 519-531.

Vaz, A.M.S.F. \& Tozzi, A.M.G.A. 2003. Bauhinia ser. Cansenia (Leguminosae: Caesalpinioideae) no Brasil. Rodriguésia 54(83): 55-143.

Vaz, A.M.S.F. \& Tozzi, A.M.G.A. 2005. Sinopse de Bauhinia sect. Pauletia (Cav.) DC. (Leguminosae: Caesalpinioideae: Cercideae) no Brasil. Revista Brasileira de Botânica 28(3): 477-491.

Velloso, A.L.; Sampaio, E.V.S.B. \& Pareyn, F.G.C. 2002. Ecorregiões propostas para o Bioma Caatinga. Recife, Associação de Plantas do Nordeste, Instituto de Conservação Ambiental The Nature Conservancy do Brasil.

Vogel, S. 1997. Remarkable nectaries: structure, ecology, organophyletic perspectives. I. Substitutive nectaries. Flora 192(4): 305-333.

Versão eletrônica do artigo em www.scielo.br/abb e http://www.botanica.org.br/acta/ojs 